\title{
Regulator of Calcineurin 1 in Periodontal Disease
}

\author{
Ulrike Peters, ${ }^{1}$ Eleni Solominidou, ${ }^{1}$ Yüksel Korkmaz, ${ }^{2}$ Stefan Rüttermann, ${ }^{3}$ Astrid Klocke, \\ Thomas Frank Flemmig, ${ }^{4}$ and Thomas Beikler ${ }^{1}$
}

${ }^{1}$ Section of Periodontics, Heinrich-Heine University, 40225 Düsseldorf, Germany

${ }^{2}$ Institute for Experimental Dental Research and Oral Musculoskeletal Biology, Center for Biochemistry, University of Cologne, 50931 Cologne, Germany

${ }^{3}$ Department of Operative Dentistry, Center for Dentistry and Oral Medicine (Carolinum), Goethe-University Frankfurt, 60596 Frankfurt, Germany

${ }^{4}$ Faculty of Dentistry, The University of Hong Kong, Sai Ying Pun, Hong Kong

Correspondence should be addressed to Thomas Beikler; beikler@med.uni-duesseldorf.de

Received 15 March 2016; Revised 20 April 2016; Accepted 5 May 2016

Academic Editor: Mirella Giovarelli

Copyright (C) 2016 Ulrike Peters et al. This is an open access article distributed under the Creative Commons Attribution License, which permits unrestricted use, distribution, and reproduction in any medium, provided the original work is properly cited.

\begin{abstract}
Nuclear factor of activated T-cells (NFAT) and NF-kB pathway associated processes are involved in the pathogenesis of various inflammatory disorders, for example, periodontal disease. The activation of these pathways is controlled by the regulator of calcineurin 1 (RCAN1). The aim of this study was to elucidate the role of RCAN1 in periodontal disease. Healthy and inflamed periodontal tissues were analyzed by immunohistochemistry and immunofluorescence using specific rabbit polyclonal anti-RCAN1 antibodies. For expression analysis human umbilical vein endothelial cells (HUVEC) were used. HUVEC were incubated for $2 \mathrm{~h}$ with Vascular Endothelial Growth Factor (VEGF) or with wild type and laboratory strains of Porphyromonas gingivalis ( $P$. gingivalis). Expression analysis of rcan 1 and $\operatorname{cox} 2$ was done by real time PCR using specific primers for rcan1.4 and cox 2 . The expression of rcanl was found to be significantly suppressed in endothelial cells of chronically inflamed periodontal tissues compared to healthy controls. Rcan 1 and cox 2 were significantly induced by VEGF and wild type and laboratory P. gingivalis strains. Interestingly, the magnitude of the rcanl and cox 2 induction was strain dependent. The results of this study indicate that RCAN1 is suppressed in endothelial cells of chronically inflamed periodontal tissues. During an acute infection, however, rcanl seems to be upregulated in endothelial cells, indicating a modulating role in immune homeostasis of periodontal tissues.
\end{abstract}

\section{Introduction}

Periodontitis is a chronic inflammatory disease resulting in the destruction of periodontal tissues and, if left untreated, in tooth loss. It is well accepted that dysbiotic microbial communities within the oral cavity are involved in the onset and progression of periodontal diseases $[1,2]$. These communities display synergistic virulence that can evade the host immune response and trigger tissue-destructive inflammatory and immune responses [3]. Many of these processes are under control of the nuclear factor of activated T-cells (NFAT) $[4,5]$ and the NF-kB pathway [6-10]. NFAT activation induces the expression of various cytokines, including IL-2, IL-3, IL-4, IL5 , IL-6, TNF- $\alpha$, and GM-CSF, whereas induction of NF-kB results in an increased expression of inflammatory cytokines like IL-1b, IL-6, TNF- $\alpha$, and IL-8 [9].
The regulator of calcineurin 1 (RCAN1) regulates NFAT and NF-kB depending pathways. The human RCAN1 genes are expressed as two isoforms, that is, RCAN1.1 and RCAN1.4 $[11,12]$. While RCAN1.1 seems to be constitutively expressed in most tissues, transcription of the RCAN1.4 variant is induced de novo by several stimuli [13]. RCAN1 interacts with cytosolic calcineurin to inhibit its phosphatase activity and thus the dephosphorylation, nuclear translocation, and activation of NFAT $[5,14,15]$. In addition to that RCAN1 led to enhanced stability of a family of NF-kB inhibitory molecules thus suppressing the NF-kB pathway $[9,16]$. RCAN1 is highly expressed in various tissues of the human body, including heart, lung, kidney, brain, muscle, liver, and testis [9]. Furthermore, it has been implicated in important physiological and pathological processes, including cell growth and immune regulation [13]. 
The human rcan1 gene is located within the Down Syndrome critical region on chromosome 21 and is overexpressed in individuals with trisomy 21 [17]. This overexpression has been implicated to mediate some of the infectious complications associated with this syndrome $[9,17]$. In this regard it is noteworthy that severe periodontitis is a common manifestation among subjects with Down Syndrome, with an estimated prevalence of 58-96\% in those under 35 years of age [18]. Moreover, the expression of rcanl has also been found to be upregulated in periodontal tissues following mechanical stress and nonsurgical periodontal therapy [19, 20], indicating a role in homoeostasis of periodontal tissues.

Taken together these findings suggest that RCAN1 is involved in the pathogenesis of periodontal diseases. Therefore, the aim of the present study was to further assess the potential role of RCAN1 in periodontal tissues by histological and coincubation studies.

\section{Materials and Methods}

2.1. Tissue Samples Collection and Tissue Preparation. Healthy $(n=6)$ and $(n=6)$ third molars with chronic periodontitis that had to be extracted for orthodontic/ medical reasons were included in the study. The patients agreed to have the tissue biopsies taken and examined for research purposes. Procurement of human teeth tissues at surgery was approved by the "Ethikkommission an der Medizinischen Fakultät der Heinrich-Heine-Universität Düsseldorf" (institutional review board of the HeinrichHeine-University Düsseldorf; IRB approval number 2980). The patients agreed to have the extracted teeth examined for research purposes. The molars and the adherent periodontal ligament (PDL) were immersion-fixed in a fixative $(4 \%$ paraformaldehyde and $0.2 \%$ picric acid in $0.1 \mathrm{M}$ phosphate buffer saline (PBS), $\mathrm{pH}$ 7.4) and demineralized for 21 days in $4 \mathrm{~N}$ formic acid. The samples were cryoprotected, frozen embedded, and frozen-sectioned on a cryostat at $30 \mu \mathrm{m}$ sections. To determine the health and inflammation state of the teeth, sections were characterized by Haematoxylin and Eosin (H\&E) staining as previously described [21].

\subsection{Immunohistochemistry}

2.2.1. Avidin-Biotin-Peroxidase Staining. Sections of healthy and inflamed molars were incubated for $48 \mathrm{~h}$ at $4^{\circ} \mathrm{C}$ with rabbit polyclonal anti-RCAN1. Then, sections were incubated with biotin-conjugated goat anti-rabbit IgG (1:500) and followed by avidin-biotin-peroxidase complex (Vector, Burlingame, CA; $1: 100$ ). The signal was visualized with $0.05 \%$ 3,3'-diaminobenzidine tetrahydrochloride (Sigma-Aldrich, Taufkirchen, Germany). Incubations without the primary antisera were carried out as immunohistochemical controls [21].

2.2.2. Double-Immunofluorescence and Confocal Microscopy. The free-floating sections were incubated with endothelial cell marker mouse anti-CD31 (1: 800) for $24 \mathrm{hrs}$ at $4^{\circ} \mathrm{C}$. Then, sections were incubated at 1:500 dilution with DyLight ${ }^{\mathrm{TM}}$ 488-conjugated goat anti-mouse IgG (Pierce Biotechnol., Rockford, IL) for $1 \mathrm{~h}$ at RT and with rabbit anti-RCAN1.4 (Santa Cruz, Heidelberg, Germany) for $24 \mathrm{hrs}$ at $4^{\circ} \mathrm{C}$. Thereafter, the sections were incubated with DyLight 549conjugated goat anti-rabbit IgG (Pierce; 1:500) for $1 \mathrm{~h}$ at RT. The nuclei were stained for $15 \mathrm{~min}$ with the DNA stain DRAQ5 (Axxora, Lörrach, Germany) at RT. The control sections were incubated without mouse anti-CD31 and rabbit anti-RCAN1.4 but with all reagents used in the immunohistochemical incubations [21]. Three colour fluorescent images were acquired on LSM 510 META confocal microscope (Carl Zeiss, Jena, Germany). The confocal images through regions of interest in each preparation at $0.1 \mu \mathrm{m}$ intervals throughout the depth of the section were collected for each fluorochrome at each z-step as described previously [21].

\subsubsection{Densitometry of the Immunohistochemical Stainings.} The densitometric analysis was performed with a Zeiss Axioskop-2 Plus microscope at 100x magnification coupled with Image System Analysis, Axiovision Ver. 4.7 (Carl Zeiss, Jena, Germany). G-intensities of RCAN1 in endothelium of blood vessels of the healthy and inflamed periodontal ligament were measured by grey values of immunostaining. The background grey value was measured from three selected regions at a section-free area. The blood vessels grey values were measured from three selected areas of the blood vessels. Immunostaining intensity was presented as the mean of measured blood vessels grey value minus mean of measured background grey value [22].

2.3. Cell Culture. Human umbilical vein endothelial cells (HUVEC) were purchased (Promocell, Heidelberg, Germany) and grown in Endothelial Cell Growth Medium (Promocell, Heidelberg, Germany) at $37^{\circ} \mathrm{C}$ in a humidified atmosphere of $95 \%$ air and $5 \% \mathrm{CO}_{2}$ in $75 \mathrm{~cm}^{2}$ flask until $70-90 \%$ confluence. The cells were harvested and seeded in tissue culture 6-well plates (Sarstedt, Germany) with counts of 60.000 cells/plate and used for experiments when having reached $80 \%$ confluence. Cells were used for experiments between passages 3 and 6 . Cell viability was determined by Trypan Blue exclusion test.

2.4. Bacterial Culture. Wild type Porphyromonas gingivalis ( $P$. gingivalis) were isolated from patients with chronic periodontitis. Type strain P. gingivalis DSM 20709 was obtained from the German Collection of Microorganisms and Cell Cultures Inc. (DSMZ Braunschweig, Germany). All bacterial strains were grown in liquid media containing $10 \%$ FCS, $3 \%$ TSB, $0.5 \%$ yeast, $0.05 \%$ L-cystein, $0.0005 \%$ hemin, and $0.001 \%$ vitamin $\mathrm{K} 1$ (all from Merck, Germany), in an anaerobic chamber (Meintrup, Germany) at an atmosphere of $80 \%$ $\mathrm{N}_{2}, 10 \% \mathrm{H}_{2}$, and $10 \% \mathrm{CO}_{2}$. All stocks were grown to OD 0.5 , centrifuged, and resuspended in an equal volume of Endothelial Cell Growth Medium (Promocell, Heidelberg, Germany).

2.5. RCAN1 and COX2 Expression Assays. HUVEC in 6well plates grown to $80 \%$ confluence were incubated with $1 \mathrm{~mL}$ bacterial suspension OD 0.5 or with $25 \mathrm{ng} / \mathrm{mL}$ Vascular 


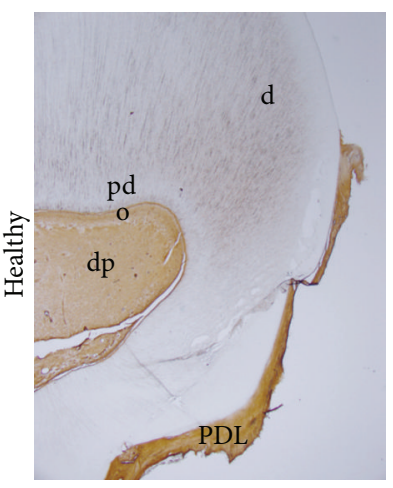

(a)

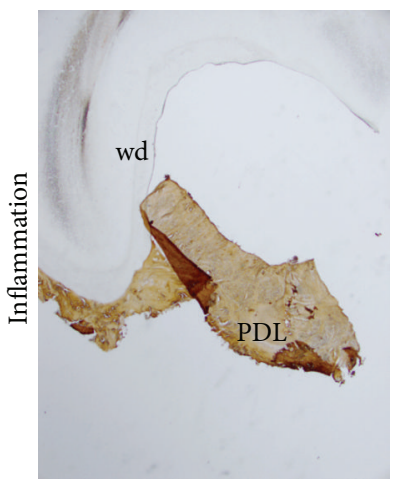

(e)

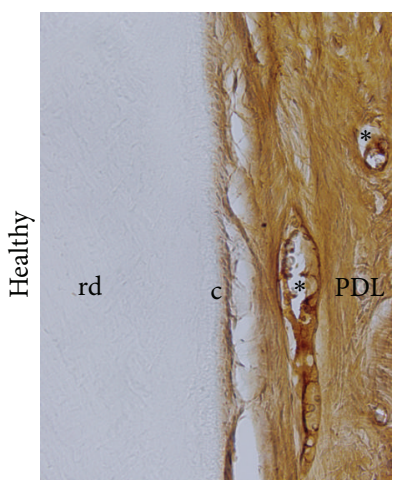

(b)

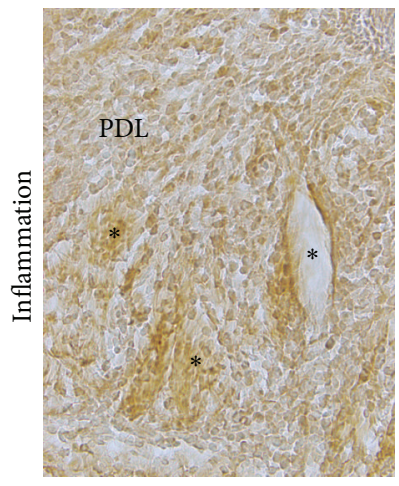

(f)

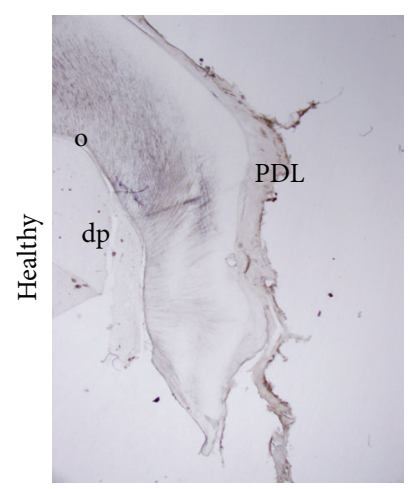

(c)

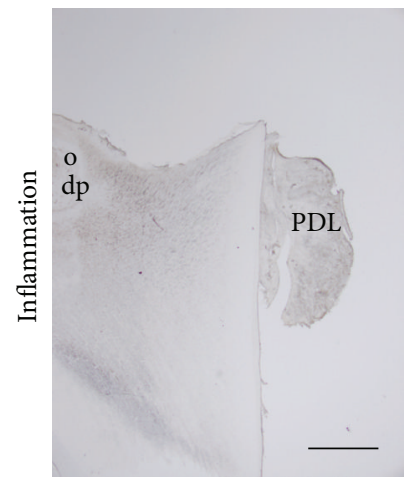

(g)

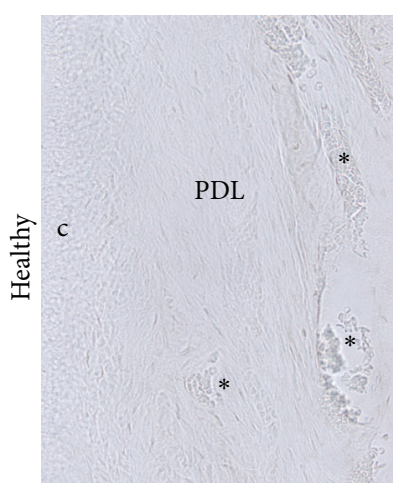

(d)

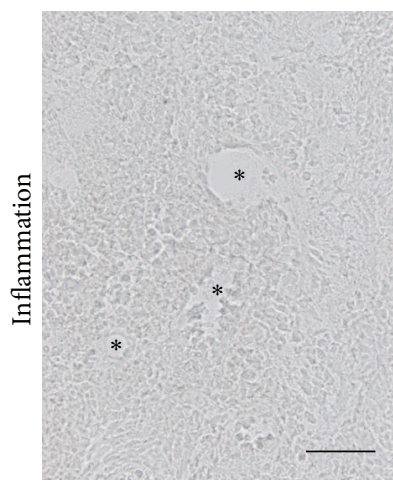

(h)

FIGURE 1: Localization of RCAN1 in healthy and chronically inflamed periodontal ligament $(\mathrm{PDL}) . \mathrm{d}=\mathrm{dentin}, \mathrm{pd}=$ predentin, $\mathrm{o}=$ odontoblasts, $\mathrm{dp}=$ dental pulp, $\mathrm{rd}=$ reactive dentin, $\mathrm{PDL}=$ periodontal ligament, and $\mathrm{c}=$ cementum. Asterisks indicate blood vessels $(\mathrm{b})$ or negative erythrocytes indicating blocked endogenous peroxidase in blood vessels (d). Bars: (a, c, e, g) $1 \mathrm{~mm}$; (b, d, f, h) $50 \mu \mathrm{m}$.

Endothelial Growth Factor (VEGF, Invitrogen Inc., Carlsbad, USA) or medium only for control. All plates were incubated for $2 \mathrm{~h}$ at $37^{\circ} \mathrm{C}$ in a humidified atmosphere of $95 \%$ air and $5 \% \mathrm{CO}_{2}$. Following incubation cells were washed with HepesBSS, detached with Detach Kit (Promocell, Heidelberg, Germany) and total RNA was isolated with Qiagen Qiashredder and Qiagen RNeasy Mini Kit according to manufacturer's instructions (Qiagen, Germany). Quantity and quality check of RNA was performed with Agilent Bioanalyzer. All experiments were run in triplicate.

2.6. Real Time PCR. Complementary DNA was synthesized using iScript cDNA Synthesis Kit (Bio-Rad, Germany). Quantitative real time PCR was performed with $2.5 \mathrm{ng} / \mu \mathrm{L}$ cDNA for gapdh and $10 \mathrm{ng} / \mu \mathrm{L}$ cDNA for rcanl in a BioRad CFX-96 Real Time System using SsoFast Eva Green Supermix (Bio-Rad, Germany). The qRT-PCR conditions were $95^{\circ} \mathrm{C}$ for $3 \mathrm{~min}$ followed by 40 cycles of $95^{\circ} \mathrm{C}$ for $10 \mathrm{~s}, 55^{\circ} \mathrm{C}$ for $5 \mathrm{~s}, 60^{\circ} \mathrm{C}$ for $10 \mathrm{~s}$, and $77^{\circ} \mathrm{C}$ for $1 \mathrm{~s}$. To verify that a specific product was amplified, a melting curve was generated at the end of PCR. Gene specific primers used were GAPDH f114 (5'-GAGTCAACGGATTTGGTCGT$\left.3^{\prime}\right)$, GAPDH r260 (5'-GACAAGCTTCCCGTTCTCAG$\left.3^{\prime}\right)$, and RCAN1 3r99 (5'-GCTCTTAAAATACTGAAAGGTG-3') according to Yao and Duh [23], and RCAN 3F ( $5^{\prime}$-TGACTGCGTGGGTCTGTAGCGC- $\left.{ }^{\prime}\right)$, COX2 exF1
( $5^{\prime}$-GCCTGGTCTGATGATGTATG-3 $\left.{ }^{\prime}\right)$, and exR ( $5^{\prime}$-GGGTAATTCCATGTTCCAGC- $3^{\prime}$ ) designed for the present study and specific for RCAN1.4 and COX2, respectively. PCR products were verified by sequencing. Gene expression level relative to gapdh and control was calculated as $\Delta \Delta$ Cq with the Bio-Rad CFX Manager 2.0 software (Bio-Rad, Germany).

2.7. Statistical Analysis. The statistical comparisons of the densitometric measurements between healthy and inflamed tissues were performed using Kruskal-Wallis test followed by two-tailed Student's $t$-test for paired samples. Differences in the expression pattern between HUVEC control and HUVEC test groups and between rcan 1 and cox 2 were analyzed by two-tailed Student's $t$-test for unpaired samples (SPSS, v.18, Munich, Germany). The significance level was set at $p<0.05$.

\section{Results}

3.1. Localization of RCAN1 in Healthy and Chronically Inflamed Periodontal Ligament (PDL). RCAN1 was detected in blood vessels of the healthy PDL (Figure 1(a)). In higher magnification of the overview picture, blood vessels (asterisks) were stained by RCAN1 (Figure 1(b)). After incubation without the primary antibodies, secondary antibodies revealed no staining (Figure 1(c)). In higher magnification of 


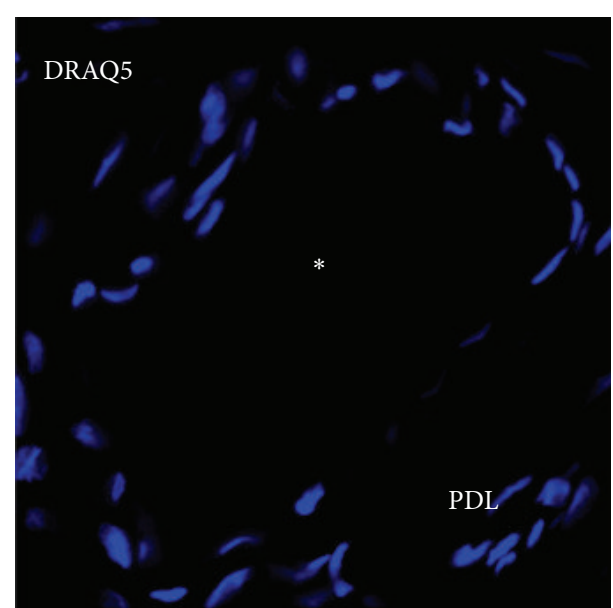

(a)

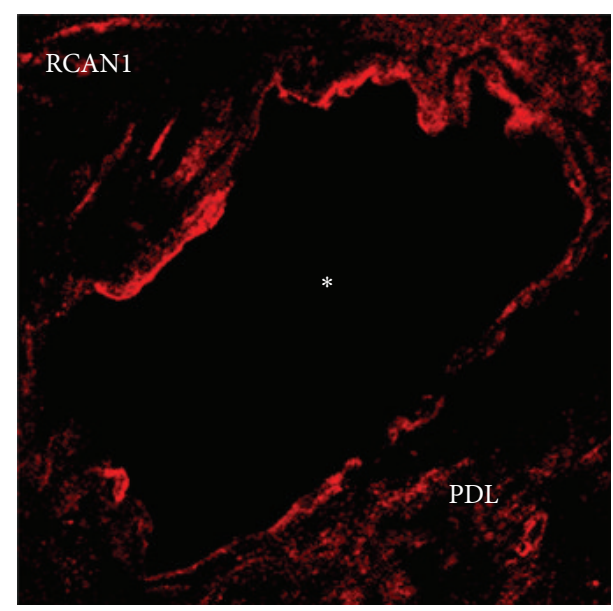

(c)

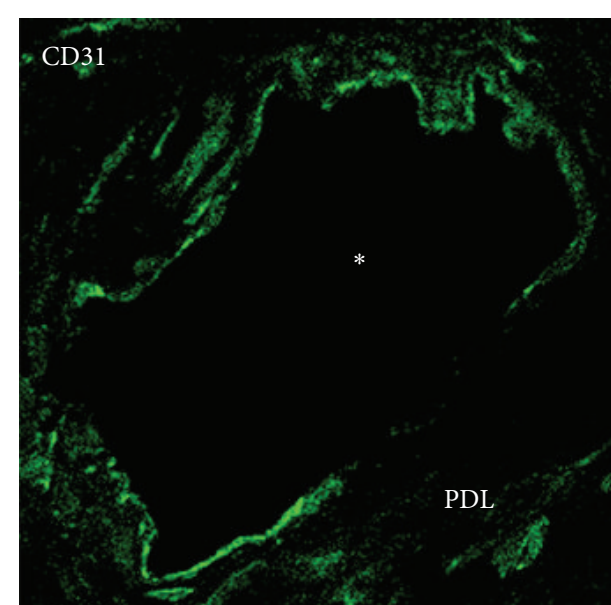

(b)

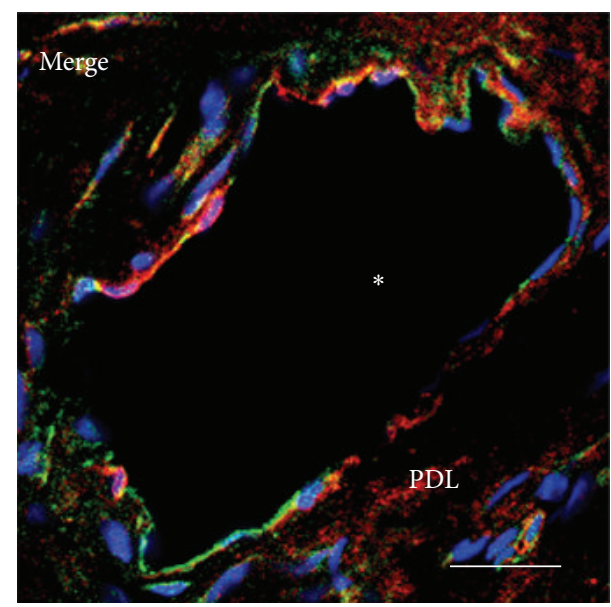

(d)

FIGURE 2: Confocal microscopy and immunofluorescence of RCAN1 in PDL. Cell nuclei of the PDL cells were identified by DRAQ5 (a; blue). The colocalization of CD31 (b: green; a marker for endothelial cells) and RCAN1 (c; red) in blood vessel walls of healthy PDL indicates that RCAN1 is localized in blood vessel endothelia. * = lumen of blood vessel (d; merge; yellow); white bar $=20 \mu \mathrm{m}$.

c, negative erythrocytes (asterisks) indicated blocked endogenous peroxidase in blood vessels of the PDL (Figure 1(d)).

In inflamed PDL, staining intensity for RCAN1 was lower in blood vessels (asterisks) when compared with healthy PDL (Figures 1(e) and 1(f)). Numerous inflammatory cells were found in the PDL (Figure 1(f)). Incubation without the primary antibodies but with secondary antibodies only revealed no staining in the inflamed control sections (Figures $1(\mathrm{~g})$ and $1(h)$ ). In inflamed PDL, negative erythrocytes (asterisks) indicated blocked endogenous peroxidase in blood vessels (Figure 1(h)).

Densitometric analysis confirmed the significantly lower staining intensity for RCAN1 in endothelial cells of the inflamed (863.23 \pm 49.60 densitometric unit) compared to endothelial cells of the healthy PDL (1422.42 \pm 90.65 densitometric unit) (Figure 3).

Confocal microscopy revealed expression of RCAN1 in endothelial cells of PDL by colocalization of CD31 and RCAN1. No colocalization with DRAQ5 and RCAN1 could be detected, indicating that RCAN1 is typically not detectable in the nuclei of PDL cells (Figures 2(a)-2(d)).

\subsection{Expression of Rcan1 and Cox 2 in Endothelial (HUVEC)} Cells. The incubation with VEGF for $2 \mathrm{~h}$ resulted in a significantly $(p<0.05) 3.56 \pm 0.33$-fold increased expression of rcanl compared to the untreated control (see Figure 4). In addition, wild type $P$. gingivalis significantly $(p<0.05)$ increased the expression of rcan1 $2.02 \pm 0.16$ to $3.64 \pm$ 0.35 -fold compared to untreated controls. Incubation with the laboratory strain DSM 20709 induced a $5.9 \pm 1.56$-fold increase in expression compared to untreated controls. The increase was found to be significantly $(p<0.05)$ increased compared to expression of rcanl induced by wild type strains of $P$. gingivalis as well as to the untreated control.

VEGF induced a significant $2.5 \pm 0.1$-fold increased $(p<$ $0.05)$ expression of $\operatorname{cox} 2$ compared to the untreated control (see Figure 5). The VEGF induced expression of cox 2 was 


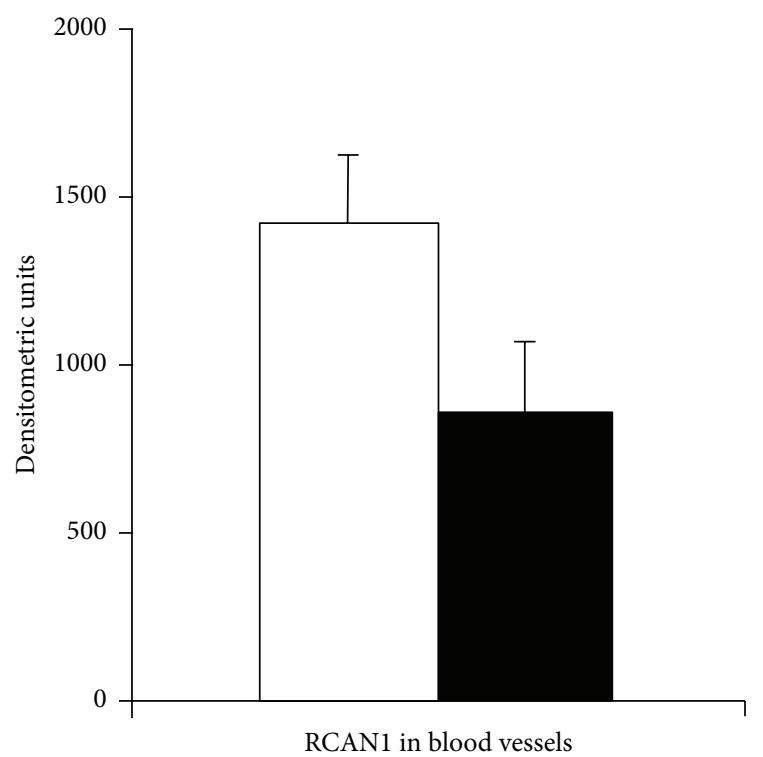

Healthy PDL

Inflamed PDI

FIGURE 3: Densitometric analysis of RCAN1 in endothelial cells. Staining intensities (densitometric units) for RCAN1 in healthy and inflamed endothelial cells. Data are mean \pm SD.

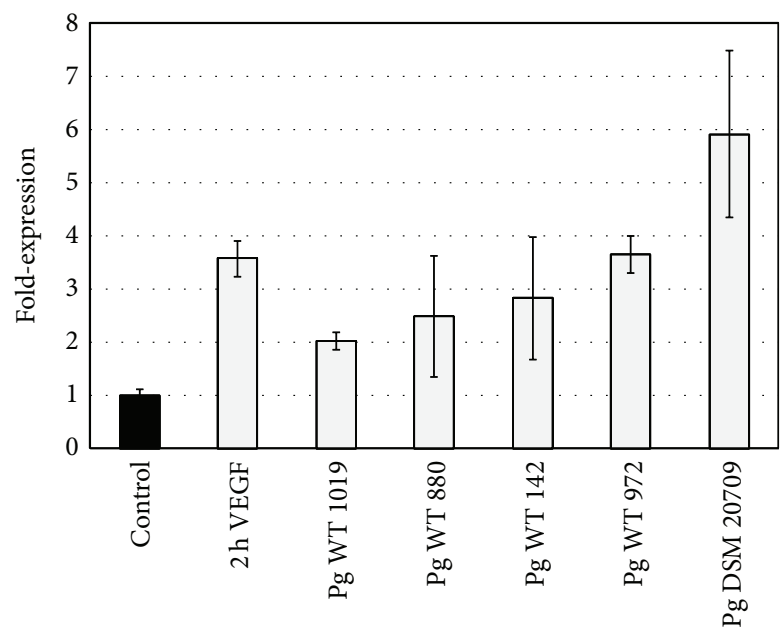

FIgURE 4: Expression of rcanl in endothelial (HUVEC) cells. Expression of rcan 1 following $2 \mathrm{~h}$ incubation with medium (negative control), VEGF (positive control), and P. gingivalis strains DSM 20709, WT 149, WT 880, WT 972, and WT 1019. Error bars indicate \pm SD.

found to be significantly lower than the VEGF induced expression of rcanl.

Wild type $P$. gingivalis significantly $(p<0.05)$ increased the expression of cox2 in a range from $1.65 \pm 0.15$ - to $4.32 \pm 0.18$-fold compared to the untreated controls. The cox 2 expression pattern induced by wild type $P$. gingivalis strains was found to be similar and not statistically different from the rcanl expression.

Incubation with laboratory strain DSM 20709 resulted in a $5.01 \pm 1.05$-fold increased expression of cox 2 compared to

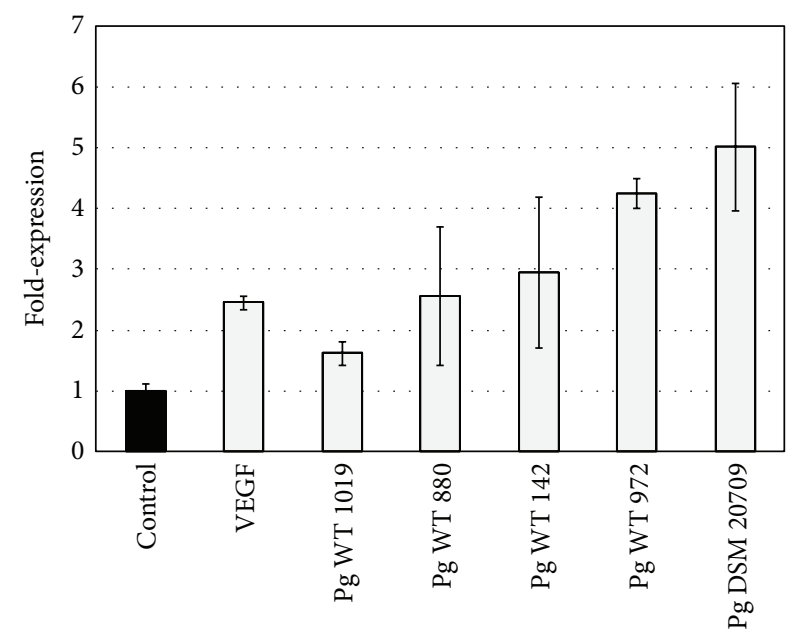

FIGURE 5: Expression of cox 2 in endothelial (HUVEC) cells. Expression of $\operatorname{cox} 2$ following $2 \mathrm{~h}$ incubation with medium (negative control), VEGF (positive control), and P. gingivalis strains DSM 20709, WT 149, WT 880, WT 972, and WT 1019. Error bars indicate \pm SD.

untreated controls. This increase was found to be significantly $(p<0.05)$ increased compared to the cox2 induced expression by wild type strains of $P$. gingivalis as well as to the untreated control. Compared to the rcanl expression, however, the P. gingivalis DSM 20709 induced cox 2 expression was found to be significantly lower.

\section{Discussion}

To our knowledge, this is the first reported histological evidence of RCAN1 expression in endothelial cells of periodontal tissues. Rcanl is a VEGF target gene in endothelial cells that regulates NFAT $[5,14,15]$ and NF-kB dependent pathways $[9,16]$ as well as other inflammatory mediators like COX2, $\mathrm{PGE}_{2}$, and thromboxane $[10,15]$. These are signaling pathways and molecules that regulate the inflammatory response in periodontal tissues.

RCAN1 has been found as both an inhibitor [24-26] and an activator [27-29] of inflammation. The apparently paradoxical actions of RCAN1 can be attributed to RCAN1's unique function in a negative feedback loop that regulates its own expression [30] as well as the activity of calcineurin $[5,10,15]$. Low or moderate levels of RCAN1 upregulate and high levels of RCAN1 downregulate calcineurin signaling suggesting that RCAN1 oscillates between stimulatory and inhibitory forms depending on its concentration [14, 29, 31]. The functional role of RCAN1 may change in a dosedependent fashion but in the opposite direction to the aforementioned studies. It has been reported that RCAN1 had an inhibitory effect at low levels but an activating effect at high levels [32]. The biological activity of rcanl appears to be highly cell and context dependent [5]. The functional RCAN1 characteristics may be responsible for the seemingly conflicting results of the present and previous studies on the role of RCAN1 in periodontal tissues [20]. In the present study, the staining densities were found to be much lower in chronically 
inflamed compared to healthy periodontal tissues. However, a previous in vivo study has shown that the expression of rcan1 is higher in treated compared to healthy periodontal tissues [20]. Based on these results one may speculate that rcan1 is downregulated during chronic infection thus fostering the immune response and upregulated during tissue regeneration to limit the inflammatory response that may interfere with the tissue repair. Although this interpretation is vaguely defined and needs to be substantiated by additional studies, the data of the above-mentioned studies indicate that the expression of RCAN1 is at least differentially regulated in healthy as well as in untreated and periodontally affected sites, respectively.

Rcan1 expression is mediated through numerous stimuli including calcium-elevating agents and cell receptor agonists like VEGF and thrombin [33]. Moreover, expression of rcan1 can be induced by Gram-positive and Gram-negative bacteria [34]. The data of the present study show that the Gramnegative putative periodontal pathogen $P$. gingivalis increases the expression of rcanl as much as VEGF, which is one of the main regulators of rcanl. This and other results indicate that TLR4 receptors may be involved in the regulation of rcan1 [34]. Furthermore, the observed phenomenon might display a short-term mechanism that limits the early immune response towards $P$. gingivalis, thus avoiding any collateral damage due to an overreacting inflammation.

Upon stimulation with VEGF and different $P$. gingivalis strains the expression of cox 2 followed almost the same pattern compared to that of rcanl, indicating a common mechanism that controls the expression of both genes. This finding is supported by other studies demonstrating that cox 2 and rcan1.4 are both upregulated by calcineurin-dependent calcium signals [35, 36]. Mobilization of intracellular calcium has been described to strongly augment the promoter activity and mRNA and protein expression of rcan1.4 and cox2 [35]. For both genes, the calcium signal component has been further found to be dependent on calcineurin and is replicated by exogenous expression of a constitutively active NFAT, strongly suggesting that the calcium-induced gene activation is mediated by NFAT $[35,37]$.

It is presently thought that RCAN1 regulation of calcineurin activity can be exploited to treat various inflammatory diseases. With regard to periodontal inflammation it is noteworthy that tacrolimus, a synthetic calcineurin inhibitor like RCAN1, has been found to exert protective effects on periodontal disease progression $[38,39]$ indicating that "fine tuning" of the NFAT-RCAN1 negative feedback loops may modulate inflammatory process in periodontitis.

In conclusion, the data of the present study provide the first further evidence that RCAN1 may be involved in modulating inflammation in a dependence of inflammation stages manner and homeostasis in periodontal ligament. However, additional studies in an animal model of periodontitis, for example, RCAN1 knockout mice, are needed to substantiate the importance of RCAN1 in periodontal disease.

\section{Competing Interests}

The authors declare no competing interests.

\section{Acknowledgments}

The authors are indebted to Claudia Heier for her invaluable technical assistance.

\section{References}

[1] G. Hajishengallis and R. J. Lamont, "Beyond the red complex and into more complexity: the polymicrobial synergy and dysbiosis (PSD) model of periodontal disease etiology," Molecular Oral Microbiology, vol. 27, no. 6, pp. 409-419, 2012.

[2] T. F. Flemmig and T. Beikler, "Control of oral biofilms," Periodontology 2000, vol. 55, no. 1, pp. 9-15, 2011.

[3] E. Gemmell and G. J. Seymour, "Immunoregulatory control of Th1/Th2 cytokine profiles in periodontal disease," Periodontology 2000, vol. 35, pp. 21-41, 2004.

[4] P. G. Hogan, L. Chen, J. Nardone, and A. Rao, “Transcriptional regulation by calcium, calcineurin, and NFAT," Genes and Development, vol. 17, no. 18, pp. 2205-2232, 2003.

[5] T. Minami, "Calcineurin-NFAT activation and DSCR-1 autoinhibitory loop: how is homoeostasis regulated?" Journal of Biochemistry, vol. 155, no. 4, pp. 217-226, 2014.

[6] B. A. Perrino, L. Y. Ng, and T. R. Soderling, "Calcium regulation of calcineurin phosphatase activity by its B subunit and calmodulin. Role of the autoinhibitory domain," The Journal of Biological Chemistry, vol. 270, pp. 340-346, 1995.

[7] J. J. Fuentes, L. Genescà, T. J. Kingsbury et al., "DSCR1, overexpressed in Down syndrome, is an inhibitor of calcineurinmediated signaling pathways," Human Molecular Genetics, vol. 9, no. 11, pp. 1681-1690, 2000.

[8] M.-G. Pan, Y. Xiong, and F. Chen, "NFAT gene family in inflammation and cancer," Current Molecular Medicine, vol. 13, no. 4, pp. 543-554, 2013.

[9] R. D. Junkins, A. J. MacNeil, Z. Wu, C. McCormick, and T.-J. Lin, "Regulator of calcineurin 1 suppresses inflammation during respiratory tract infections," Journal of Immunology, vol. 190, no. 10, pp. 5178-5186, 2013.

[10] T. Minami, M. Miura, W. C. Aird, and T. Kodama, “Thrombininduced autoinhibitory factor, Down syndrome critical region1, attenuates NFAT-dependent vascular cell adhesion molecule1 expression and inflammation in the endothelium," Journal of Biological Chemistry, vol. 281, no. 29, pp. 20503-20520, 2006.

[11] K. J. A. Davies, G. Ermak, B. A. Rothermel et al., "Renaming the DSCR1/Adapt78 gene family as RCAN: regulators of calcineurin," The FASEB Journal, vol. 21, no. 12, pp. 3023-3028, 2007.

[12] J. J. Fuentes, M. A. Pritchard, and X. Estivill, "Genomic organization, alternative splicing, and expression patterns of the DSCR1 (Down Syndrome Candidate Region 1) gene," Genomics, vol. 44, no. 3, pp. 358-361, 1997.

[13] N. Méndez-Barbero, V. Esteban, S. Villahoz et al., "A major role for RCAN1 in atherosclerosis progression," EMBO Molecular Medicine, vol. 5, no. 12, pp. 1901-1917, 2013.

[14] C. D. Harris, G. Ermak, and K. J. A. Davies, "Multiple roles of the DSCR1 (Adapt78 or RCAN1) gene and its protein product calcipressin 1 (or RCAN1) in disease," Cellular and Molecular Life Sciences, vol. 62, no. 21, pp. 2477-2486, 2005.

[15] B. A. Hesser, X. H. Liang, G. Camenisch et al., "Down syndrome critical region protein 1 (DSCR1), a novel VEGF target gene that regulates expression of inflammatory markers on activated endothelial cells," Blood, vol. 104, no. 1, pp. 149-158, 2004. 
[16] Y. S. Kim, K. O. Cho, H. J. Lee, S. Y. Kim, Y. Sato, and Y. J. Cho, "Down syndrome candidate region 1 increases the stability of the I $\kappa \mathrm{B} \alpha$ protein: implications for its anti-inflammatory effects," Journal of Biological Chemistry, vol. 281, no. 51, pp. 39051-39061, 2006.

[17] K. R. Martin, D. Layton, N. Seach et al., "Upregulation of RCAN1 causes down syndrome-like immune dysfunction," Journal of Medical Genetics, vol. 50, no. 7, pp. 444-454, 2013.

[18] J. M. Albandar, "Aggressive and acute periodontal diseases," Periodontology 2000, vol. 65, no. 1, pp. 7-12, 2014.

[19] R. M. S. de Araujo, Y. Oba, and K. Moriyama, "Identification of genes related to mechanical stress in human periodontal ligament cells using microarray analysis," Journal of Periodontal Research, vol. 42, no. 1, pp. 15-22, 2007.

[20] T. Beikler, U. Peters, K. Prior, M. Eisenacher, and T. F. Flemmig, "Gene expression in periodontal tissues following treatment," BMC Medical Genomics, vol. 1, no. 1, article 30, 2008.

[21] Y. Korkmaz, H. Lang, T. Beikler et al., "Irreversible inflammation is associated with decreased levels of the $\alpha 1-, \beta 1-$, and $\alpha 2$-subunits of sGC in human odontoblasts," Journal of Dental Research, vol. 90, no. 4, pp. 517-522, 2011.

[22] Y. Korkmaz, W. Bloch, D. Steinritz et al., "Bradykinin mediates phosphorylation of eNOS in odontoblasts," Journal of Dental Research, vol. 85, no. 6, pp. 536-541, 2006.

[23] Y.-G. Yao and E. J. Duh, "VEGF selectively induces Down syndrome critical region 1 gene expression in endothelial cells: a mechanism for feedback regulation of angiogenesis?" Biochemical and Biophysical Research Communications, vol. 321, no. 3, pp. 648-656, 2004.

[24] B. Chan, G. Greenan, F. McKeon, and T. Ellenberger, "Identification of a peptide fragment of DSCR1 that competitively inhibits calcineurin activity in vitro and in vivo," Proceedings of the National Academy of Sciences of the United States of America, vol. 102, no. 37, pp. 13075-13080, 2005.

[25] S. Ryeom, R. J. Greenwald, A. H. Sharpe, and F. McKeon, "The threshold pattern of calcineurin-dependent gene expression is altered by loss of the endogenous inhibitor calcipressin," Nature Immunology, vol. 4, no. 9, pp. 874-881, 2003.

[26] Y. J. Yang, A. J. MacNeil, R. Junkins et al., "Regulator of calcineurin 1 (Rcan1) is required for the development of pulmonary eosinophilia in allergic inflammation in mice," The American Journal of Pathology, vol. 179, no. 3, pp. 1199-1210, 2011.

[27] T. J. Kingsbury and K. W. Cunningham, "A conserved family of calcineurin regulators," Genes \& Development, vol. 14, no. 13, pp. 1595-1604, 2000.

[28] B. Sanna, E. B. Brandt, R. A. Kaiser et al., "Modulatory calcineurin-interacting proteins 1 and 2 function as calcineurin facilitators in vivo," Proceedings of the National Academy of Sciences of the United States of America, vol. 103, no. 19, pp. 73277332, 2006.

[29] Z. Hilioti, D. A. Gallagher, S. T. Low-Nam et al., "GSK-3 kinases enhance calcineurin signaling by phosphorylation of RCNs," Genes and Development, vol. 18, no. 1, pp. 35-47, 2004.

[30] E. Cano, A. Canellada, T. Minami, T. Iglesias, and J. M. Redondo, "Depolarization of neural cells induces transcription of the Down syndrome critical region 1 isoform 4 via a calcineurin/nuclear factor of activated T cells-dependent pathway," The Journal of Biological Chemistry, vol. 280, no. 33, pp. 2943529443, 2005.

[31] M. M. Corsi, W. Ponti, A. Venditti et al., "Proapoptotic activated T-cells in the blood of children with Down's syndrome: relationship with dietary antigens and intestinal alterations," International Journal of Tissue Reactions, vol. 25, no. 3, pp. 117125, 2003.

[32] S.-Y. Shin, H. W. Yang, J.-R. Kim, W. D. Heo, and K.-H. Cho, "A hidden incoherent switch regulates RCAN1 in the calcineurinNFAT signaling network," Journal of Cell Science, vol. 124, no. 1, pp. 82-90, 2011.

[33] D. V. Riper, L. Jayakumar, N. Latchana et al., "Regulation of vascular function by RCAN1 (ADAPT78)," Archives of Biochemistry and Biophysics, vol. 472, no. 1, pp. 43-50, 2008.

[34] D. L. Bhoiwala, V. Kannabiran, S. F. Hushmendy et al., "The calcineurin inhibitor RCAN1 is involved in cultured macrophage and in vivo immune response," FEMS Immunology and Medical Microbiology, vol. 61, no. 1, pp. 103-113, 2011.

[35] A. Canellada, B. G. Ramirez, T. Minami, J. M. Redondo, and E. Cano, "Calcium/calcineurin signaling in primary cortical astrocyte cultures: Rcanl-4 and cyclooxygenase- 2 as NFAT target genes," Glia, vol. 56, no. 7, pp. 709-722, 2008.

[36] R. R. Baggott, A. Alfranca, D. López-Maderuelo et al., "Plasma membrane calcium ATPase isoform 4 inhibits vascular endothelial growth factor-mediated angiogenesis through interaction with calcineurin," Arteriosclerosis, Thrombosis, and Vascular Biology, vol. 34, no. 10, pp. 2310-2320, 2014.

[37] K. Holmes, E. Chapman, V. See, and M. J. Cross, "Vegf stimulates RCAN1.4 expression in endothelial cells via a pathway requiring $\mathrm{Ca}^{2+} /$ calcineurin and protein kinase $\mathrm{C}-\delta$," PLoS ONE, vol. 5, no. 7, article el1435, 2010.

[38] D. C. Andia, C. A. Nassar, P. O. Nassar, M. R. Guimarães, P. S. Cerri, and L. C. Spolidorio, "Treatment with tacrolimus enhances alveolar bone formation and decreases osteoclast number in the maxillae: a histomorphometric and ultrastructural study in rats," Histology and Histopathology, vol. 23, no. 10, pp. 1177-1184, 2008.

[39] M. R. Guimarães, P. O. Nassar, D. C. Andia et al., "Protective effects of Tacrolimus, a calcineurin inhibitor, in experimental periodontitis in rats," Archives of Oral Biology, vol. 52, no. 9, pp. 882-888, 2007. 


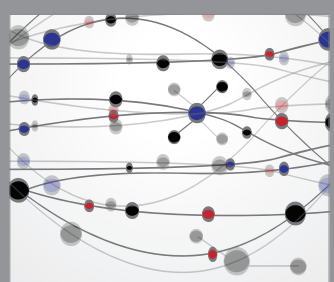

The Scientific World Journal
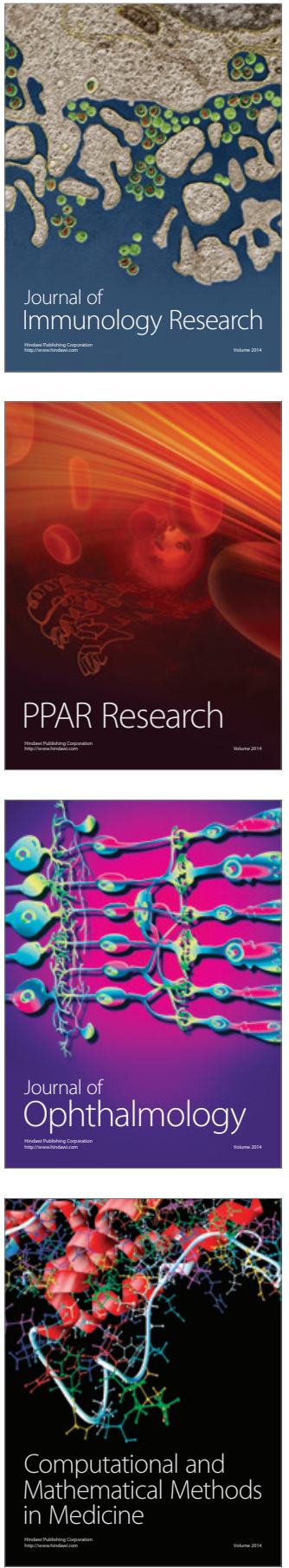

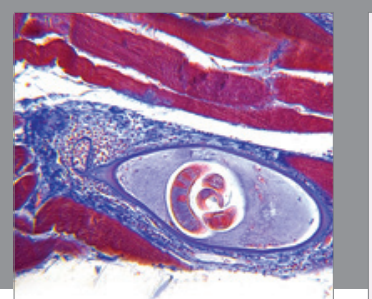

Gastroenterology Research and Practice

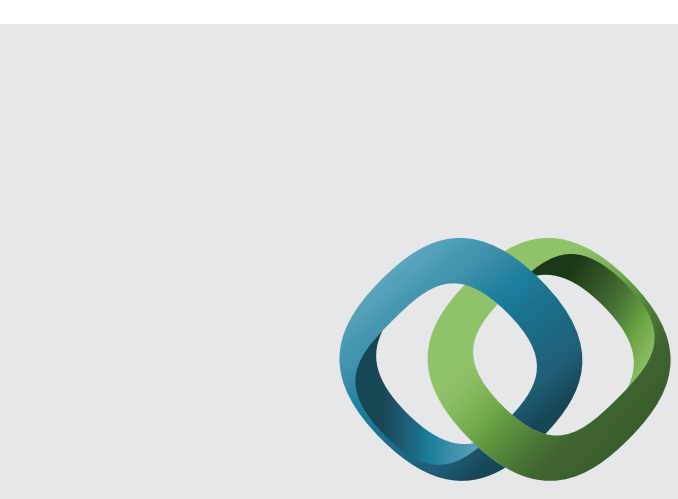

\section{Hindawi}

Submit your manuscripts at

http://www.hindawi.com
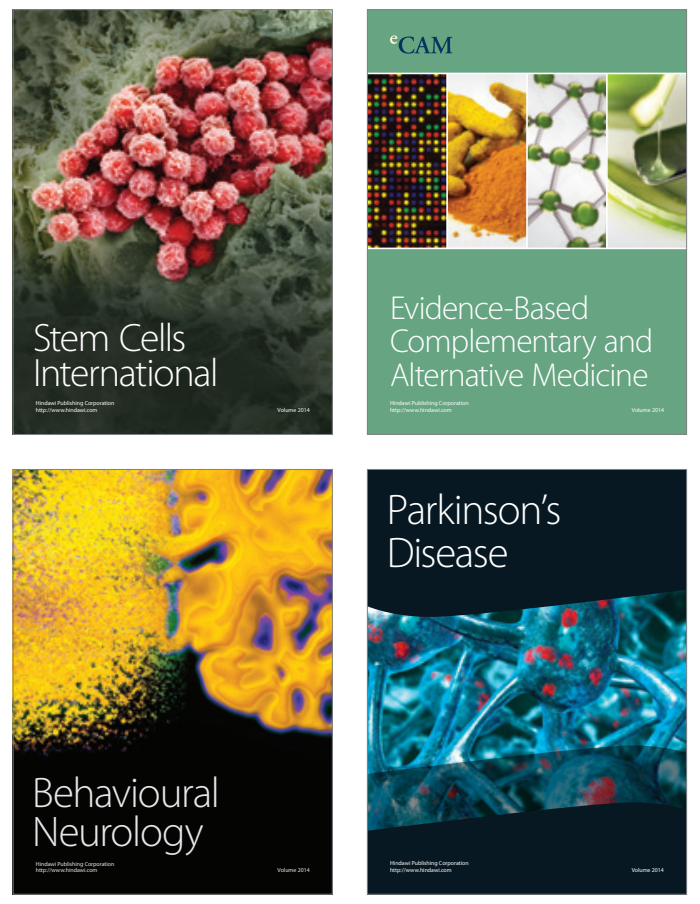
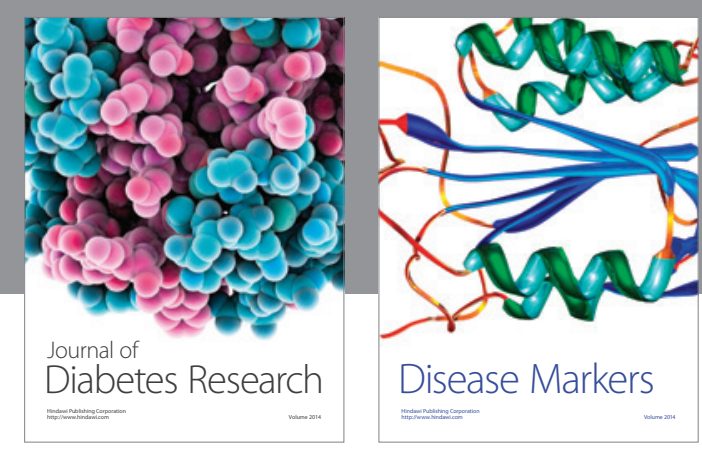

Disease Markers
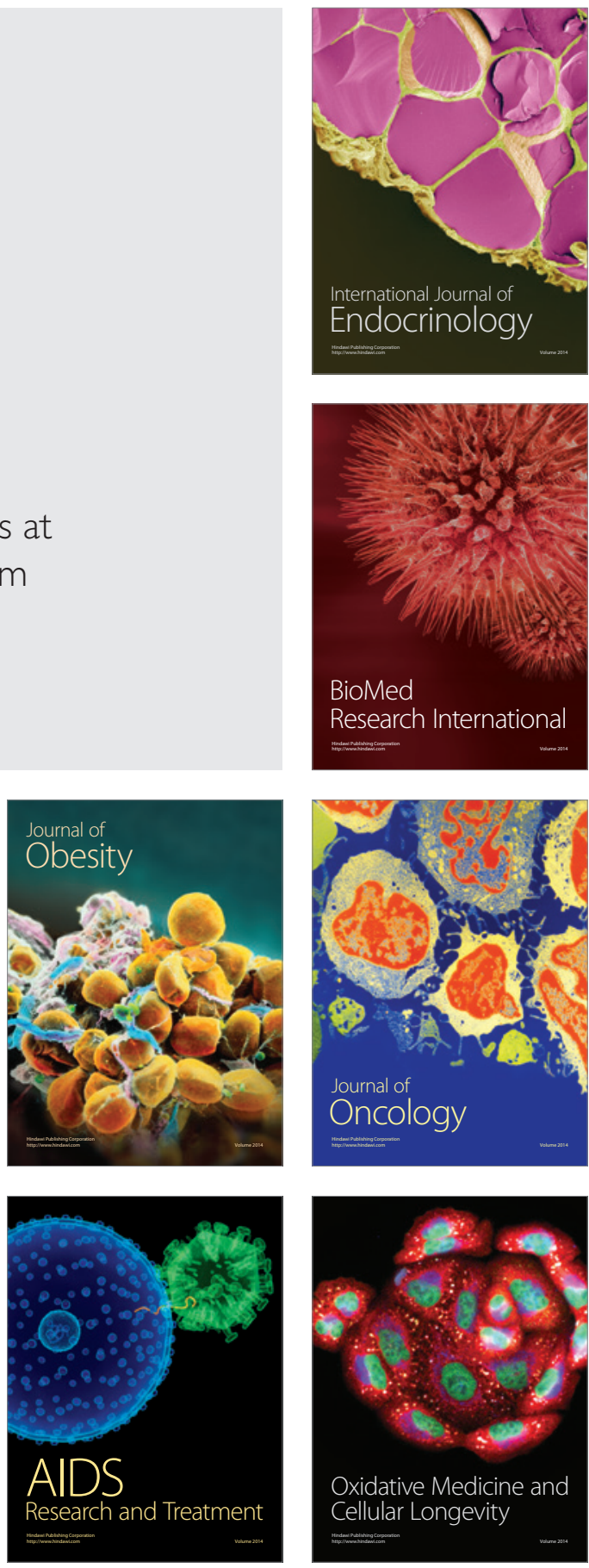